\title{
DECOMPOSIÇÃO E LIBERAÇÃO DE NITROGÊNIO, FÓSFORO E POTÁSSIO DE RESÍDUOS DA CULTURA DO FEIJOEIRO ${ }^{(1)}$
}

\author{
Eduardo Chagas ${ }^{(2)}$, Adelson Paulo Araújo ${ }^{(3)}$, Marcelo Grandi Teixeira ${ }^{(4)}$ \& \\ José Guilherme Marinho Guerra ${ }^{(5)}$
}

\begin{abstract}
RESUMO
Este trabalho teve como objetivo mensurar a velocidade de decomposição e de liberação de nutrientes in situ de resíduos da cultura do feijoeiro (Phaseolus vulgaris L.). Em condições de campo, foram coletadas as folhas senescentes durante o ciclo da cultura, bem como os caules e a palhada de vagens após trilhagem dos grãos, de cultivares de feijoeiro. Esses resíduos continham 2,3 $\mathrm{Mg} \mathrm{ha}^{-1} \mathrm{de}_{\text {massa, }}$ $31 \mathrm{~kg} \mathrm{ha}^{-1}$ de $\mathrm{N}$ e $2,4 \mathrm{~kg} \mathrm{ha}^{-1}$ de $\mathrm{P}$, que correspondiam a 63,41 e $28 \%$ do total de massa, $\mathbf{N}$ e $\mathrm{P}$, respectivamente, acumulados durante o ciclo da cultura. Foram realizados dois ensaios, nos períodos de inverno-primavera e primavera-verão, quando os caules, as vagens e as folhas senescentes foram colocados separadamente em litterbags, dispostos sobre o solo, e coletados entre 2 e 120 dias. As quantidades de matéria seca e nutrientes remanescentes em cada tipo de resíduo foram ajustadas a um modelo exponencial simples. O tempo de meia-vida dos resíduos foi de, no primeiro e segundo ensaios, respectivamente, 133 e 179 dias para caules, 70 e 80 dias para folhas e 64 dias para vagens. A relativamente lenta decomposição dos resíduos pode ser associada à sua alta relação $\mathrm{C}: \mathrm{N}$ - acima de 60 para caule e palhada de vagens. A liberação de $\mathrm{N}$ e $\mathrm{P}$ pelos resíduos foi similar à dinâmica de decomposição, enquanto a liberação de $\mathrm{K}$ foi mais rápida. Os resultados indicam que as folhas senescentes e os resíduos produzidos após trilhagem dos grãos podem restituir parte da demanda de nutrientes do feijoeiro, assumindo relevância para sistemas de agricultura sustentável.
\end{abstract}

Termos de indexação: matéria orgânica, ciclagem de nutrientes, feijão, Phaseolus vulgaris.

\footnotetext{
(1) Recebido para publicação em dezembro de 2005 e aprovado em abril de 2007.

(2) Engenheiro-Agrônomo do Instituto de Defesa Agropecuária e Florestal do Espírito Santo - IDAF. Escritório de Rio Bananal, CEP 29920-000 Rio Bananal (ES). E-mail: parbananal@idaf.es.gov.br

(3) Professor do Departamento de Solos da Universidade Federal Rural do Rio de Janeiro - UFRRJ. CEP 23890-000 Seropédica (RJ). Bolsista do CNPq. E-mail: aparaujo@ufrrj.br

(4) Pesquisador da Embrapa Agrobiologia. CEP 23890-000, Seropédica (RJ). E-mail: grandi@cnpab.embrapa.br

(5) Pesquisador da Embrapa Agrobiologia. Bolsista do CNPq. E-mail: gmguerra@cnpab.embrapa.br
} 


\title{
SUMMARY: DECOMPOSITIONAND RELEASE OF NITROGEN, PHOSPHORUS AND POTASSIUM FROM RESIDUES OF COMMON BEAN CROP
}

\begin{abstract}
The objective of this study was to measure the speed of decomposition and nutrient release in situ from residues of common bean (Phaseolus vulgaris L.) crop. In field conditions, leaves of common bean cultivars senesced during the growth cycle and stems and podwalls after pod threshing were collected. These residues contained $2.3 \mathrm{Mg} \mathrm{ha}{ }^{-1}$ of mass, $31 \mathrm{~kg} \mathrm{ha}^{-1}$ of $N$ and $2.4 \mathrm{~kg} \mathrm{ha}^{-1}$ of $P$ that corresponded to 63,41 and $28 \%$ of total mass, $N$ and $P$, respectively accumulated during the crop growth cycle. Two experiments were conducted, during winter-spring and spring-summer. Stems, podwalls and senesced leaves were placed separately in litterbags on the ground and collected, from the $2^{\text {nd }}$ to the $120^{\text {th }}$ day. The dry matter and nutrient amounts found in each residue type were adjusted to a simple exponential model. The half-life of the residues was, in the first and second trial respectively, 133 and 179 days for stems, 70 and 80 days for leaves, and 64 days for podwalls. The relatively slow residue decomposition may be associated with the high $C: N$ ratio $(C: N>60$ in stems and podwalls). Nitrogen and P release by residues was similar to the decomposition pattern, while K release was faster. The results indicate that senescent leaves and residues produced after pod threshing can cover part of the nutrient demand of common bean crop, which is relevant for sustainable agricultural systems.
\end{abstract}

Index terms: organic matter, nutrient cycling, Phaseolus vulgaris.

\section{INTRODUÇÃO}

A maximização da ciclagem de nutrientes, pelo adequado manejo dos resíduos vegetais produzidos num cultivo, é uma opção para aumentar a sustentabilidade dos sistemas agrícolas, otimizando seus recursos internos. Quantificar a contribuição dos resíduos dos cultivos no fornecimento de nutrientes pode introduzir novas práticas de manejo, que podem otimizar a ciclagem de nutrientes e reduzir a demanda por insumos externos. Os resíduos de cultivos de leguminosas, dependendo do tipo e da qualidade do material, contêm, usualmente, de 20 a $80 \mathrm{~kg} \mathrm{ha}^{-1}$ de $\mathrm{N}$, mas em alguns casos podem conter acima de $150 \mathrm{~kg} \mathrm{ha}^{-1}$ de N (Shah et al., 2003). Os resíduos resultantes da trilhagem de grãos de cultivares de feijoeiro (Phaseolus vulgaris L.) apresentaram entre 0,6 e $1,5 \mathrm{Mg}^{-1}$ de matéria seca, e continham entre 5 e $12 \mathrm{~kg} \mathrm{ha}^{-1}$ de $\mathrm{N}$ e entre 0,4 e $1,0 \mathrm{~kg} \mathrm{ha}^{-1}$ de $\mathrm{P}$, o que correspondia em média a 15 e $11 \%$ do total de $\mathrm{N}$ e $\mathrm{P}$, respectivamente, acumulados pela cultura quando da maturação dos grãos (Araújo \& Teixeira, 2003). A substituição das práticas de queima de resíduos por seu adequado manejo pode diminuir a emissão de gases para a atmosfera, reduzindo os impactos da agricultura no efeito estufa, além de incorporar quantidades substanciais de matéria orgânica ao solo (Lal, 2003).

A decomposição dos resíduos dos cultivos depende da natureza e da quantidade do material vegetal, da fertilidade do solo, do grau de fracionamento do resíduo, além das condições climáticas, representadas principalmente pelo regime de chuvas e temperatura, que influenciam a atividade microbiana do solo (Bertol et al., 2004). Entre as características do material orgânico que afetam a dinâmica do processo de decomposição, podem-se citar o teor de nutrientes, lignina e polifenóis, a relação $\mathrm{C}: \mathrm{N}$, a resistência física e o tamanho do material (Tian et al., 1992; Aita \& Giacomini, 2003). Os três principais métodos utilizados na medição da decomposição de resíduos vegetais são a respiração do solo (indicada pela evolução do $\mathrm{CO}_{2}$ após incubação dos resíduos no solo), a relação entre a quantidade do material que cai do dossel e a que está depositada sobre o solo e as avaliações diretas, por meio de medidas de perda de massa em sacos de tela para o estudo da decomposição, ou litterbags (Correia \& Andrade, 1999).

A introdução de leguminosas em sistemas de rotação de culturas tem importância não apenas no fornecimento de $\mathrm{N}$ aos cultivos subseqüentes, mas também a longo prazo, por aumentar os estoques de nutrientes como $\mathrm{N}$ e $\mathrm{P}$ e contribuir com o aumento do teor de matéria orgânica do solo (Ladd et al., 1981). Cerca de 72 a $77 \%$ do ${ }^{15} \mathrm{~N}$ adicionado por resíduos de alfafa (Medicago littoralis) permaneceu como N orgânico no solo após cultivo subseqüente de trigo (Ladd et al., 1981), e, em média, $46 \%$ do ${ }^{15} \mathrm{~N}$ originalmente adicionado por resíduos de alfafa (Medicago sativa) permaneceu no solo após cultivo subseqüente de milho (Harris \& Hesterman, 1990). Leguminosas perenes aumentaram o conteúdo de $\mathrm{P}$ na forma de diésteres no solo, incrementando a disponibilidade de $\mathrm{P}$ em formas orgânicas lábeis (Canellas et al., 2004). Entretanto, os benefícios de cultivos de leguminosas de grão só podem ser efetivamente logrados caso os resíduos dos cultivos sejam mantidos no solo: em experimentos de rotação no Paquistão, os ingressos de $\mathrm{N}$ 
variaram, respectivamente com ou sem a retenção dos resíduos, entre 112 e $74 \mathrm{~kg} \mathrm{ha}^{-1}$ de $\mathrm{N}$ para o mungo (Vigna radiata) e 64 e $9 \mathrm{~kg} \mathrm{ha}^{-1}$ de $\mathrm{N}$ para a lentilha (Lens culinaris) (Shah et al., 2003).

O feijoeiro tem sido considerado uma espécie com baixa capacidade de nodulação e fixação biológica de $\mathrm{N}_{2}$, quando comparado a outras leguminosas de grão (Graham et al., 2003). Apesar disso, a fixação do $\mathrm{N}_{2}$ contribuiu com até $90 \mathrm{~kg}$ ha-1 de $\mathrm{N}$ em vários cultivos de feijão, o que representou de 40 a 50 \% da demanda dessa cultura (Westermann et al., 1981), e em sete experimentos de campo foram observados valores médios e máximos de, respectivamente, 35 e $70 \%$ do $\mathrm{N}$ derivado da atmosfera (Hardarson et al., 1993). Mesmo que a maior parte do $\mathrm{N}$ obtido pela fixação biológica de $\mathrm{N}_{2}$ seja removida nos grãos colhidos de feijão, as folhas senescentes depositadas durante o ciclo da cultura e os resíduos obtidos após a trilhagem dos grãos podem fornecer quantidades de nutrientes, que vão restituir ao solo parte da demanda da cultura ou fornecer nutrientes para cultivos posteriores em sistemas de rotação de culturas. Na colheita mecanizada do feijão, os resíduos da parte aérea da cultura mantêm-se sobre o solo até sua completa decomposição, enquanto nas colheitas manual e semimecanizada a parte aérea é arrancada e transportada para locais onde os grãos são trilhados (Silva \& Fonseca, 1996). Para que sejam adequadamente manejados, é necessário demonstrar aos agricultores que esses resíduos apresentam valor como fonte de matéria orgânica e nutrientes para o crescimento de cultivos subseqüentes (Shah et al., 2003). Entretanto, há poucas informações sobre a quantidade de nutrientes acumulados nos resíduos da cultura do feijoeiro, bem como sobre a dinâmica de liberação de nutrientes ao solo pela sua decomposição.

Este trabalho teve como objetivo mensurar a velocidade de decomposição e de liberação de N, P e K de resíduos da cultura de feijoeiro.

\section{MATERIAL E MÉTODOS}

Em condições de campo na área experimental da Embrapa Agrobiologia (Seropédica-RJ), foram cultivados quatro cultivares de feijoeiro entre junho e outubro de 2002, em 16 parcelas de $30 \mathrm{~m}^{2}$. Em cada parcela foram delimitadas duas áreas de $0,5 \mathrm{~m}^{2}$, onde foram coletadas as folhas que se desprenderam das plantas, duas vezes por semana, sendo esse material reunido, seco em estufa e pesado. Quando da maturação de grãos, as plantas da área de $1 \mathrm{~m}^{2}$ no centro de cada parcela foram colhidas, as vagens foram trilhadas, as sementes pesadas, e os caules e palhada das vagens, secos e pesados. Os resíduos produzidos apresentavam em média massa de $2,3 \mathrm{Mg} \mathrm{ha}^{-1}$ e continham $31 \mathrm{~kg} \mathrm{ha}^{-1}$ de $\mathrm{N}$ e $2,4 \mathrm{~kg} \mathrm{ha}^{-1}$ de $\mathrm{P}$, o que correspondeu a 63,41 e $28 \%$ do total de massa, $\mathrm{NeP}$, respectivamente, acumulados pela cultura durante seu ciclo (Quadro 1).

Esses resíduos foram utilizados em ensaios para determinação de sua velocidade de decomposição quando em contato com o solo, por meio de litterbags. Considerando que a dinâmica de decomposição de misturas de folhas e caules assume padrões diferentes da decomposição de folhas e caules isoladamente (Quemada \& Cabrera, 1995), foram analisados separadamente os caules, a palhada das vagens e as folhas senescentes dos quatro cultivares. Foram utilizados sacos de polivinil (litterbags) com malha de $4 \mathrm{~mm}$ e área de $25 \times 25 \mathrm{~cm}$ e $1,5 \mathrm{~cm}$ de altura. Esse formato evita a compressão das folhas dentro dos sacos e permite o acesso da maioria dos organismos decompositores (Tian et al., 1992). Cada material foi caracterizado quanto ao teor de $\mathrm{C}$, por incineração em forno de mufla; ao teor de N, pelo método semimicro Kjeldahl; e ao teor de P, por digestão nitro-perclórica e dosagem colorimétrica; no extrato nitro-perclórico foi dosado o teor de $\mathrm{K}$, por fotometria de chama (Malavolta et al., 1989) (Quadro 2).

Foram realizados dois ensaios de campo, o primeiro entre julho e novembro de 2003, com os resíduos das cultivares Xamego e Carioca, e o segundo entre outubro de 2003 e janeiro de 2004, com os resíduos das cultivares Manteigão e Ouro Negro, que correspondem, aproximadamente, ao período após a colheita dos plantios da "seca" na região Centro-Sul do Brasil. Foram colocados $15 \mathrm{~g}$ de caule, $15 \mathrm{~g}$ de vagem e $5 \mathrm{~g}$ de folha senescente, em seu tamanho original, sem qualquer trituração, em cada saco plástico separadamente; a menor massa inicial de folhas senescentes foi conseqüência da dificuldade de

Quadro 1. Matéria seca e conteúdos de nitrogênio e fósforo em caules, palhada de vagens e grãos na maturação, e em folhas senescentes coletadas durante o ciclo da cultura de feijoeiro (médias de quatro cultivares)

\begin{tabular}{lccc}
\hline & Caule & Palhada de vagem & Folha senescente \\
\hline Massa $\left(\mathrm{kg} \mathrm{ha}^{-1}\right)$ & 810 & 432 & 1.032 \\
Conteúdo de $\mathrm{N}\left(\mathrm{kg} \mathrm{ha}^{-1}\right)$ & 5,8 & 4,1 & 21,3 \\
Conteúdo de P $\left(\mathrm{kg} \mathrm{ha}^{-1}\right)$ & 0,46 & 0,25 & 4,66 \\
\hline
\end{tabular}


Quadro 2. Teores de carbono, nitrogênio, fósforo e potássio em caules, palhada de vagens e folhas senescentes de feijoeiro, utilizados nos ensaios de velocidade de decomposição (médias de quatro cultivares)

\begin{tabular}{|c|c|c|c|c|c|}
\hline Resíduo & Teor de C & Teor de N & Teor de $\mathbf{P}$ & Teor de K & Relação C:N \\
\hline & $\mathrm{kg} \mathrm{kg}^{-1}$ & 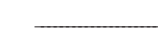 & $\mathrm{g} \mathrm{kg}^{-1}$ & $\underline{-}$ & \\
\hline Caules & 0,54 & 6,8 & 0,49 & 15,3 & 79 \\
\hline Palhada de vagens & 0,53 & 8,0 & 0,50 & 33,1 & 66 \\
\hline Folhas senescentes & 0,48 & 20,4 & 1,54 & 9,3 & 24 \\
\hline
\end{tabular}

obtenção de quantidades apreciáveis deste resíduo. No primeiro ensaio, os sacos plásticos foram dispostos aleatoriamente na superfície do solo, presos ao solo com vergalhões de metal e coletados aos $2,5,8,12$, 16,30 e 60 dias para folhas, e até 120 dias para caule e vagem após sua instalação. No segundo ensaio, os sacos foram coletados aos 2, 4, 8, 16, 32 e 64 dias para folhas e até 110 dias para caule e vagem, com três repetições. A temperatura média durante o período de condução do primeiro e segundo ensaios foi de, respectivamente, 22,1 e $24,8^{\circ} \mathrm{C}$. No primeiro ensaio, a precipitação total foi de $470 \mathrm{~mm}$, e apenas $27 \%$ desse total ocorreu na primeira metade de duração do ensaio; no segundo ensaio, a precipitação total atingiu $595 \mathrm{~mm}$, com $57 \%$ desse total ocorrendo na primeira metade do ensaio.

Em cada época de coleta, o material foi retirado dos sacos, removendo-se possíveis elementos de contaminações por lavagem superficial, seco em estufa a $65^{\circ} \mathrm{C}$, pesado e moído, sendo determinados os teores de N, de $\mathrm{P}$ e de K (Malavolta et al., 1989). O conteúdo de cada nutriente foi obtido pelo produto entre o teor e a matéria seca. Para avaliar a presença de eventuais contaminações de solo, em amostras de folha, caule e palhada de vagens coletadas no tempo zero e após 16 e 110 dias da instalação dos ensaios, foi determinada a proporção de cinzas, incinerando-se 2,0 g de amostra em forno de mufla (Amado et al., 2003).

Os dados de matéria seca e conteúdos de nutrientes remanescentes para cada tipo de resíduo foram ajustados a um modelo de decomposição descrito por uma equação exponencial simples, $x_{t}=x_{0} e^{-k t}$, em que $\mathrm{x}_{\mathrm{t}}$ é a fração da massa remanescente no tempo $t ; \mathrm{x}_{0}$, a fração de massa remanescente no tempo zero; $t$, o tempo em dias; e k, a constante de decomposição. A transformação dos dados em logaritmo natural torna o modelo uma equação de primeiro grau (Wieder \& Lang, 1982). A meia-vida $\left(t_{1 / 2}\right)$ de cada resíduo, que expressa o tempo necessário para que metade do material inicial se decomponha, foi obtida por $\mathrm{t}_{1 / 2}=0,693 / \mathrm{k}$. Os valores de constante de decomposição de cada material foram comparados estatisticamente por meio de um teste $t$, considerando seus respectivos erros-padrões (Neter et al., 1990).

\section{RESULTADOS E DISCUSSÃO}

No primeiro ensaio, o material de vagem foi perdido devido ao ataque de formigas, e são apresentados apenas os resultados referentes a caule e folha. As proporções de cinzas após incineração em mufla foram similares nas diferentes épocas de amostragem - em torno de 16, 7 e $9 \%$ para folhas senescentes, caules e palhada de vagens, respectivamente - , indicando pequena contaminação com solo dos materiais coletados; assim, optou-se por não efetuar qualquer correção de massa no material remanescente (Amado et al., 2003). Não foram identificadas diferenças significativas entre cultivares na velocidade de decomposição dos resíduos, sendo, portanto, efetuado um ajuste dos dados médios das duas cultivares em cada ensaio. O modelo exponencial simples ajustado aos dados de massa remanescente mostrou-se adequado, com elevados coeficientes de correlação, todos significativos a 0,1 \% pelo teste t (Quadro 3). Esperava-se que a decomposição desses resíduos fosse relativamente rápida; logo, foram utilizados intervalos curtos entre as primeiras coletas, mas não foi isso o que foi observado (Figura 1). Dessa forma, algumas estimativas de meia-vida situaram-se além do tempo de duração dos ensaios, introduzindo alguma incerteza nessas estimativas (Quadro 3).

Nos dois ensaios, houve diferenças significativas entre os resíduos na velocidade de decomposição. A palhada de vagens e as folhas senescentes apresentaram velocidade de decomposição similar, e os caules foram decompostos mais lentamente (Quadro 3). Os resíduos apresentaram constante de decomposição relativamente baixa e meia-vida relativamente elevada. Para efeito de comparação, Tian et al. (1992) registraram constantes de decomposição de 0,023 dia ${ }^{-1}$ em resíduos da poda de leucena (Leucaena leucocephala), enquanto Amado et al. (2003) observaram, em resíduos de ervilhaca, constantes de decomposição de 0,054 e 0,012 dia-1 em dois diferentes anos de avaliação. Já Aita \& Giacomini (2003) observaram constantes de decomposição de 0,061 e 0,0021 dia-1, para os compartimentos facilmente decomponível e recalcitrante, respectivamente, de resíduos de ervilhaca (Vicia sativa L.). Uma das razões para essa lenta 
Quadro 3. Parâmetros do modelo exponencial simples ajustado aos dados da fração remanescente da massa e dos conteúdos de nitrogênio, fósforo e potássio, de resíduos de plantas de feijoeiro (caules, palhada de vagens e folhas senescentes), em dois ensaios de decomposição in situ com o uso de litterbags

\begin{tabular}{|c|c|c|c|c|c|c|}
\hline \multirow{2}{*}{ Resíduo vegetal } & \multicolumn{3}{|c|}{ Primeiro ensaio } & \multicolumn{3}{|c|}{ Segundo ensaio } \\
\hline & Correlação & $\begin{array}{l}\text { Constante de } \\
\text { decomposição }\end{array}$ & Meia-vida & Correlação & $\begin{array}{l}\text { Constante de } \\
\text { decomposição }\end{array}$ & Meia-vida \\
\hline & & $\mathrm{dia}^{-1}$ & dia & & $\operatorname{dia}^{-1}$ & dia \\
\hline \multicolumn{7}{|l|}{ Massa } \\
\hline Vagens & - & - & - & 0,979 & $0,010862 \mathrm{a}$ & 64 \\
\hline Caules & 0,990 & $0,005195 \mathrm{~b}$ & 133 & 0,916 & $0,003868 \mathrm{~b}$ & 179 \\
\hline Folhas & 0,984 & 0,009883 a & 70 & 0,969 & $0,008639 \mathrm{a}$ & 80 \\
\hline \multicolumn{7}{|l|}{ Conteúdo de $\mathrm{N}$} \\
\hline Vagens & - & - & - & 0,853 & $0,009113 \mathrm{ab}$ & 76 \\
\hline Caules & 0,880 & $0,005853 \mathrm{a}$ & 118 & 0,637 & $0,005237 \mathrm{~b}$ & 132 \\
\hline Folhas & 0,979 & $0,010309 \mathrm{a}$ & 67 & 0,982 & $0,012950 \mathrm{a}$ & 54 \\
\hline \multicolumn{7}{|l|}{ Conteúdo de $\mathrm{P}$} \\
\hline Vagens & - & - & - & 0,769 & $0,013658 \mathrm{ab}$ & 51 \\
\hline Caules & 0,934 & 0,009277 a & 75 & 0,623 & $0,004144 \mathrm{~b}$ & 167 \\
\hline Folhas & 0,984 & $0,009540 \mathrm{a}$ & 73 & 0,976 & $0,015121 \mathrm{a}$ & 46 \\
\hline \multicolumn{7}{|l|}{ Conteúdo de $\mathrm{K}$} \\
\hline Vagens & - & - & - & 0,761 & 0,038427 a & 18 \\
\hline Caules & 0,859 & $0,035442 \mathrm{a}$ & 20 & 0,691 & $0,031921 \mathrm{a}$ & 22 \\
\hline Folhas & 0,938 & $0,054892 \mathrm{a}$ & 13 & 0,737 & $0,041388 \mathrm{a}$ & 17 \\
\hline
\end{tabular}

No primeiro ensaio, o material de vagem foi perdido pelo ataque de formigas. Constantes de decomposição seguidas pela mesma letra, dentro de cada nutriente, não diferem entre si pelo teste t a $5 \%$; todos os coeficientes de correlação são significativos a $0,1 \%$ pelo teste $t$.
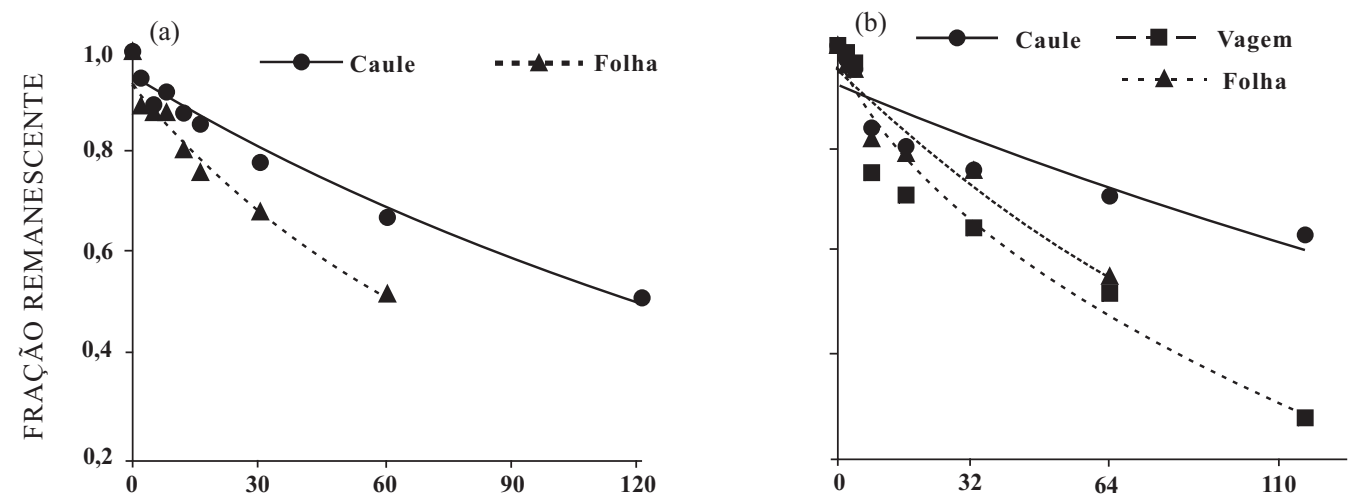

DIA

Figura 1. Fração remanescente da massa de caule, palhada de vagens e folhas senescentes de plantas de feijoeiro, em dois ensaios de decomposição in situ com o uso de litterbags; pontos representam médias experimentais, e linhas, as curvas ajustadas ao modelo exponencial simples; (a) primeiro ensaio e (b) segundo ensaio.

decomposição dos resíduos do feijoeiro foi sua relação C:N relativamente elevada, acima de 60 para caules e palhada de vagens (Quadro 2), pois materiais com relação C:N acima de 30 geralmente apresentam decomposição mais lenta (Tian et al., 1992). Apesar da menor relação $\mathrm{C}: \mathrm{N}$ das folhas senescentes, a meia- vida delas foi similar à das vagens (Quadro 3). As constantes de decomposição foram relativamente similares nos dois ensaios, uma vez que as condições de temperatura e precipitação, que podem influenciar a dinâmica de decomposição (Bertol et al., 2004), foram semelhantes nos dois períodos. 
O modelo exponencial apresentou ajuste significativo $(p<0,001)$ para os dados de conteúdo remanescente de N, P e K nos resíduos, apesar dos coeficientes de correlação inferiores aos obtidos no ajuste de massa seca (Quadro 3). Nos dois ensaios, a liberação de $\mathrm{N}$ e $\mathrm{P}$ pelos resíduos da cultura do feijoeiro foi similar à dinâmica de decomposição, sendo mais lenta que a liberação de $\mathrm{K}$, como indicado pela menor meia-vida do K para caule, folha e vagem (Quadro 3). Tian et al. (1992) também observaram que a liberação de $\mathrm{N}$ e $\mathrm{P}$ dos resíduos de leguminosas seguiu um padrão semelhante à decomposição de matéria seca, porém a liberação de $\mathrm{K}$ foi mais rápida, em virtude da maior mobilidade deste nutriente nos tecidos vegetais e de sua maior lixiviação. No primeiro ensaio, não houve diferenças significativas entre materiais nas constantes de decomposição para N, P e K. Já no segundo ensaio, os caules apresentaram liberação mais lenta de $\mathrm{N}$ e $\mathrm{P}$ do que as folhas senescentes, observando-se similaridade entre folhas senescentes e vagens (Quadro 3).

A biomassa depositada pelas folhas senescentes das quatro cultivares de feijoeiro estudadas atingiu $1,0 \mathrm{Mg} \mathrm{ha}^{-1}$ e continha em média 28 e $19 \%$ do total de $\mathrm{N}$ e $\mathrm{P}$, respectivamente, acumulados pela cultura durante seu ciclo de crescimento (Quadro 1), o que indica a grande relevância das folhas senescentes nos processos de ciclagem de nutrientes. Chagas et al. (2003) verificaram que a deposição de folhas senescidas por sete cultivares de feijoeiro foi pequena e estável até a terceira semana, com aumento a partir da quarta semana; a maior deposição de folhas senescidas ocorreu na quinta semana após a floração, período de maturação dos grãos. Considerando a meia-vida estimada para liberação dos nutrientes (Quadro 3), o $\mathrm{N}$ e o P provenientes de folhas senescentes só poderiam ser utilizados por um cultivo subseqüente, mas parte do K liberado pela decomposição das folhas senescentes, em particular das folhas depositadas nas primeiras semanas após a floração, poderia hipoteticamente ser reabsorvida no mesmo cultivo.

Os caules e a palhada de vagens produzidos após a trilhagem dos grãos das quatro cultivares estudadas atingiram massa de $1,2 \mathrm{Mg}^{-1} \mathrm{e}^{-1}$ continham 9,9 e $0,7 \mathrm{~kg} \mathrm{ha}^{-1}$ de $\mathrm{N}$ e $\mathrm{P}$, respectivamente (Quadro 1), resultados similares aos observados por Araújo \& Teixeira (2003). Os grãos, por sua vez, continham 59 e $72 \%$ do total de $\mathrm{N}$ e $\mathrm{P}$ acumulados pela cultura durante seu ciclo de crescimento (Quadro 1), indicando elevada exportação de nutrientes pelos grãos colhidos. Mesmo admitindo-se elevada estimativa de $70 \%$ do $\mathrm{N}$ derivado da fixação simbiótica (Hardarson et al., 1993), o balanço líquido de $\mathrm{N}$ deve ser negativo para a cultura do feijão, com maior quantidade de $\mathrm{N}$ exportado nos grãos do que a fixada simbioticamente durante o cultivo. Apesar da relativamente pequena quantidade de $\mathrm{N}$ e $\mathrm{P}$ presente nos caules e vagens após trilhagem dos grãos, esses resíduos continham 18 e $10 \%$ do total de $\mathrm{N}$ e $\mathrm{P}$, respectivamente, acumulados pela cultura quando da maturação fisiológica (Quadro 1). O retorno ao solo dos caules e da palhada de vagens obtidos após trilhagem dos grãos, com sua provável lenta decomposição (Quadro 3), pode reduzir as perdas de nutrientes do sistema e aumentar os estoques de $\mathrm{N}$ e P orgânicos do solo, assumindo relevância para a sustentabilidade da cultura.

\section{CONCLUSÕES}

1. As folhas senescentes depositadas durante o ciclo da cultura do feijoeiro apresentaram meia-vida de 70 e 80 dias, velocidade de decomposição similar à da palhada de vagens obtidas após trilhagem dos grãos, com meia-vida de 64 dias, por sua vez mais rápida que a decomposição dos caules, com meia-vida de 133 e 179 dias.

2. A liberação de $\mathrm{N}$ e $\mathrm{P}$ pelos resíduos da cultura do feijoeiro foi similar à dinâmica de decomposição, enquanto a liberação de $\mathrm{K}$ foi mais rápida.

\section{LITERATURA CITADA}

AITA, C. \& GIACOMINI, S.J. Decomposição e liberação de nitrogênio de resíduos culturais de plantas de cobertura de solo solteiras e consorciadas. R. Bras. Ci. Solo, 27:601$612,2003$.

AMADO, T.J.C.; SANTI, A. \& ACOSTA, J.A.A. Adubação nitrogenada na aveia preta. II - Influência na decomposição de resíduos, liberação de nitrogênio e rendimento de milho sob sistema plantio direto. R. Bras. Ci. Solo, 27:1085-1096, 2003.

ARAÚJO, A.P. \& TEIXEIRA, M.G. Nitrogen and phosphorus harvest indices of common bean cultivars: Implications for yield quantity and quality. Plant Soil, 257:425-433, 2003.

BERTOL, I.; LEITE, D. \& ZOLDAN JR., W.A. Decomposição do resíduo de milho e variáveis relacionadas. R. Bras. Ci. Solo, 28:369-375, 2004.

CANELLAS, L.P.; ESPÍNDOLA, J.A.A.; GUERRA, J.G.M.; TEIXEIRA, M.G.; VELLOSO, A.C.X. \& RUMJANEK, V.M. Phosphorus analysis in soil under herbaceous perennial leguminous cover by nuclear magnetic spectroscopy. Pesq. Agropec. Bras., 39:589-596, 2004.

CHAGAS, E.; DEL-PIN, B.; KUBOTA, F.Y.; ARAÚJO, A.P. \& TEIXEIRA, M.G. Conteúdo de nutrientes em folhas senescentes de cultivares de feijoeiro. In: CONGRESSO BRASILEIRO DE CIÊNCIA DO SOLO, 29., Ribeirão Preto, 2003. Anais. Ribeirão Preto, Universidade Estadual de São Paulo/Sociedade Brasileira de Ciência do Solo, 2003. CD-ROM

CORREIA, M.E.F. \& ANDRADE, A.G. Formação de serapilheira e ciclagem de nutrientes. In: SANTOS, G.A. \& CAMARGO, F.A.O., eds. Fundamentos da matéria orgânica do solo. Porto Alegre, Genesis, 1999. p.197-225. 
GRAHAM, P.H.; ROSAS, J.C.; DE JENSEN, C.E.; PERALTA, E.; TLUSTY, B.; ACOSTA-GALLEGOS, J. \& PEREIRA, P.A.A. Addressing edaphic constraints to bean production: The Bean/Cowpea CRSP project in perspective. Field Crops Res., 82:179-192, 2003.

HARDARSON, G.; BLISS, F.A.; CIGALES-RIVERO, M.R.; HENSON, R.A.; KIPE-NOLT, J.A.; LONGERI, L.; MANRIQUE, A.; PEÑA-CABRIALES, J.J.; PEREIRA, P.A.A.; SANABRIA, C.A. \& TSAI, S.M. Genotypic variation in biological nitrogen fixation by common bean. Plant Soil, 152:59-70, 1993.

HARRIS, G.H. \& HESTERMAN, O.B. Quantifying the nitrogen contribution from alfafa to soil and two succeeding crops using nitrogen-15. Agron. J., 82:129-134, 1990.

LADD, J.N.; OADES, J.M. \& AMATO, M. Distribution and recovery of nitrogen from legume residues decomposing in soils sown to wheat in the field. Soil Biol. Biochem., 13:251-256, 1981.

LAL, R. Global potential of soil carbon sequestration to mitigate the greenhouse effect. Critical Rev. Plant Sci., 22:151. 184, 2003.

MALAVOLTA, E.; VITTI, G.C. \& OLIVEIRA, S.A. Avaliação do estado nutricional das plantas. Piracicaba, Associação Brasileira para Pesquisa da Potassa e do Fosfato, 1989 201p.
NETER, J.; WASSERMAN, W. \& KUTNER, M.H. Applied linear statistical models. 3.ed. Homewood, Richard D. Irwin, 1990. 1181p.

QUEMADA, M. \& CABRERA, M.L. Carbon and nitrogen mineralized from leaves and stems of four cover crops. Soil Sci. Soc. Am. J., 59:471-477, 1995.

SHAH, Z.; SHAH, S.H.; PEOPLES, M.B.; SCHWENKE, G.D. \& HERRIDGE, D.F. Crop residue and fertiliser $\mathrm{N}$ effects on nitrogen fixation and yields of legume-cereal rotations and soil organic fertility. Field Crops Res., 83:1-11, 2003.

SILVA, J.G. \& FONSECA, J.R. Colheita. In: ARAUJO, R.S.; RAVA, C.A.; STONE, L.F. \& ZIMMERMANN, M.J.O., coords. Cultura do feijoeiro comum no Brasil. Piracicaba, Potafos, 1996. p.523-541.

TIAN, G.; KANG, B.T. \& BRUSSAARD, L. Biological effects of plant residues with contrasting chemical compositions under humid tropical conditions - decomposition and nutrient release. Soil Biol. Biochem., 24:1051-1060, 1992.

WESTERMANN, D.T.; KLEINKOPF, G.E.; PORTER, L.K. \& LEGGETT, G.E. Nitrogen sources for bean seed production. Agron. J., 73:660-664, 1981.

WIEDER, R.K. \& LANG, G.E. A critique of the analytical methods used in examining decomposition data obtained from litter bags. Ecology, 63:1636-1642, 1982. 